\title{
Online lab books for supervision of project students
}

\author{
Badge, J. L. ${ }^{1}$ and Badge R. M. ${ }^{2}$ \\ ${ }^{1}$ School of Biological Sciences, University of Leicester, ${ }^{2}$ Department of Genetics, University \\ of Leicester
}

Date accepted: $x x / x x / x x$

Here we report a case study where Blackboard's wiki function was used to create electronic lab books for the supervision of undergraduate students completing laboratory based research projects.

At the University of Leicester, in August 2008, Dr Richard Badge created an electronic lab book to replace his own paper lab book. Contained within a specific Blackboard module, it utilises the campus learning objects wiki tool (www.learningobjects.com/teams.jsp). Users of the wiki can create new pages, edit other user's pages and leave comments. All actions are username and date stamped. The editing tool enables simple word processing, the creation of tables and the insertion of images. The system is secure, in that it is hosted within the VLE and can be accessed only by specified University account holders. There are daily, system-wide VLE back-ups at institutional level, and weekly back-ups held locally by Dr Badge.

The wiki system provides certain functions that are either impossible or convoluted to perform with paper. For example, when re-using common protocols, either a hyperlink can be inserted to refer to a methods page or the protocol can be copied and pasted into a new page. Since all lab members can view and edit all the wiki pages, protocols and data can be easily shared online at any time.

At the University of Leicester, Biological Sciences undergraduates complete a 30-day research project in the first semester of their third year worth 40 credits. Most students undertake the laboratory research during October and November. They then give a short oral presentation in March and submit their final written reports at the beginning of May.

In 2008/09 Richard Badge supervised two undergraduate project students. The students had access to networked computers at the end of each bench. They were encouraged to plan their experiments on the wiki, write up the protocol and then print a copy to work with at the bench. Any changes to the paper protocol were transferred back to the wiki. The students undertook molecular biology projects that involved PCR genotyping, long range PCR, PCR product purification and sequencing of DNA from humans or chimpanzees. As a result much of their data comprised digital photographs of agarose gels, which could be inserted directly into wiki pages.

Analysis of the students' use of the wiki was carried out using a combination of Blackboard tracking systems and the wiki revision history. Overall, student 1 used the wiki to a much greater extent than student 2 , with double the number of recorded visits to the Blackboard site over the period 1 October 2008 to 6 May 2009. 


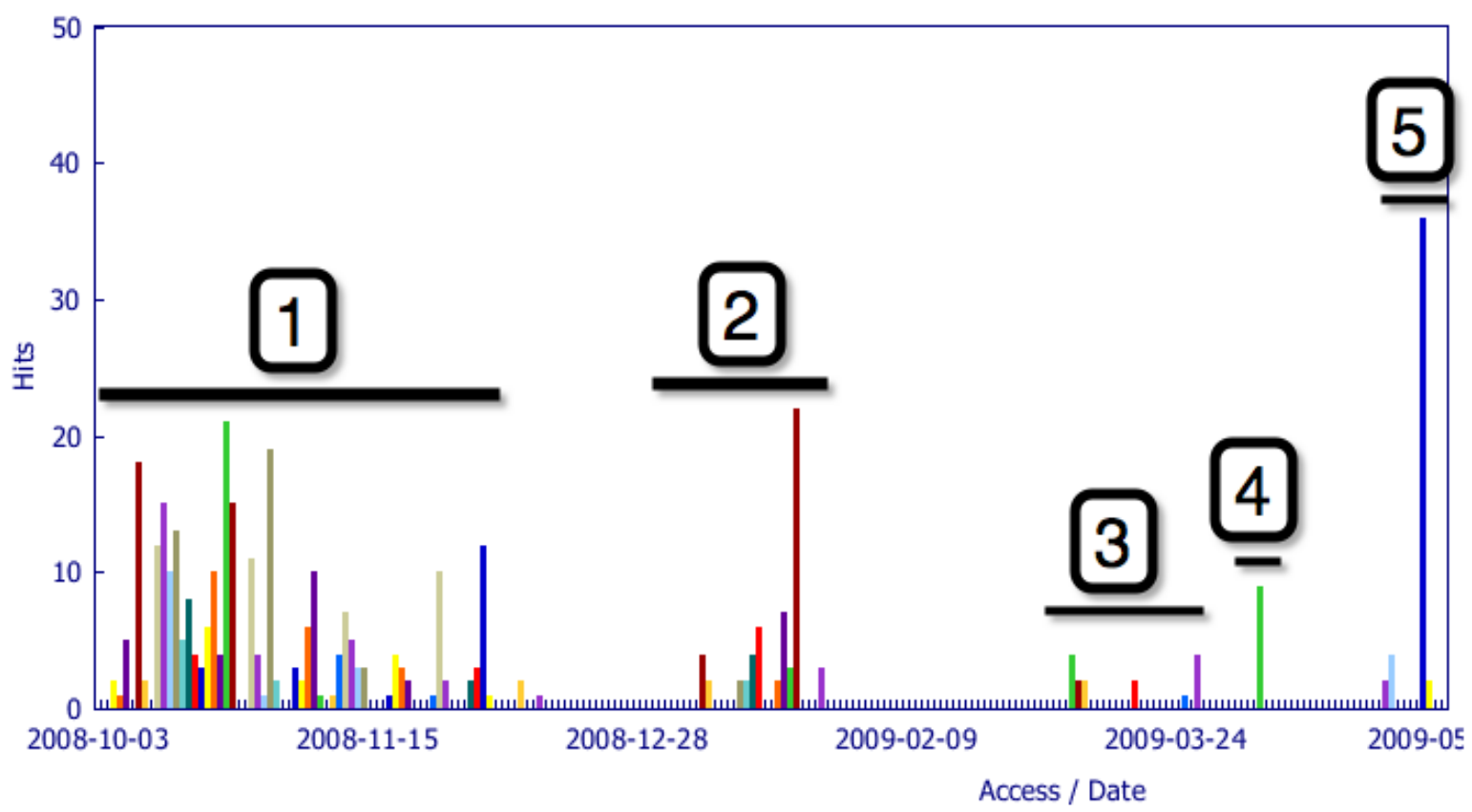

Figure 1 Student 1 blackboard access statistics from 1 October 2008 to 6 May 2009

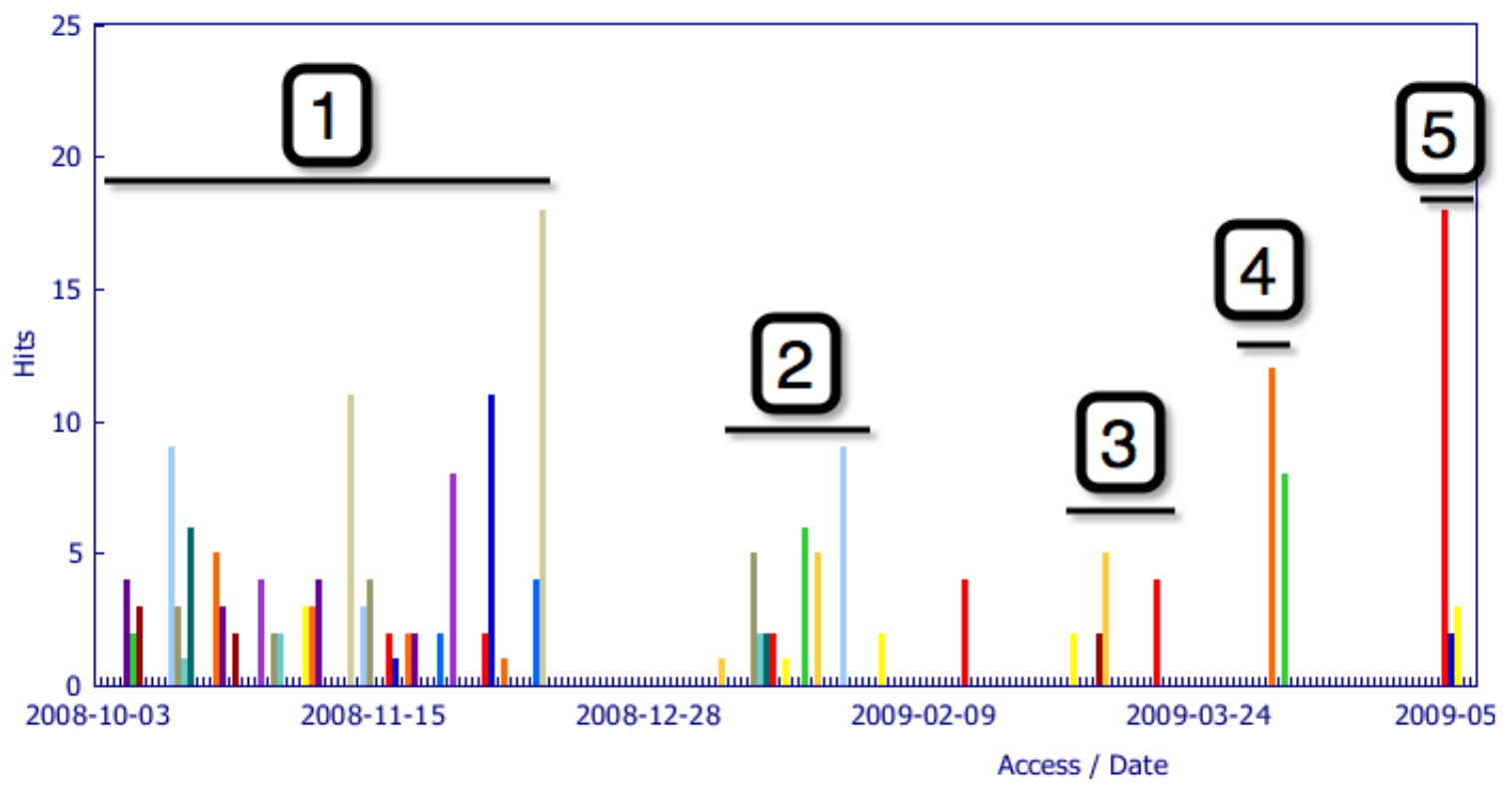

Figure 2 Student 2 blackboard access statistics from 1 October 2008 to 6 May 2009

Peaks in access can be seen during the main phases of the project (see figures 1 and 2):

1. laboratory phase of the project (October and November)

2. final tidying of data in January

3. access prior to the oral presentation in March

4. preparation of written report during April

5. final submission of the written report on 5 May

Student 1 kept a daily log of activity on the wiki and accessed the wiki when not present in the lab. Student one created 49 separate pages on 49 separate days. Student 2 worked within two pages of the wiki, adding to them 54 times on 29 separate days. 


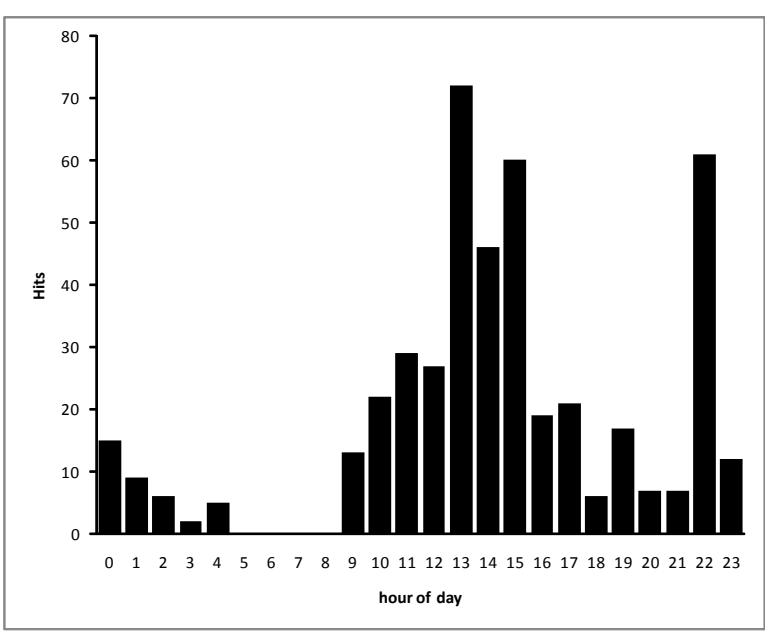

Figure 3 Time of access to lab Blackboard site by hour for student 1

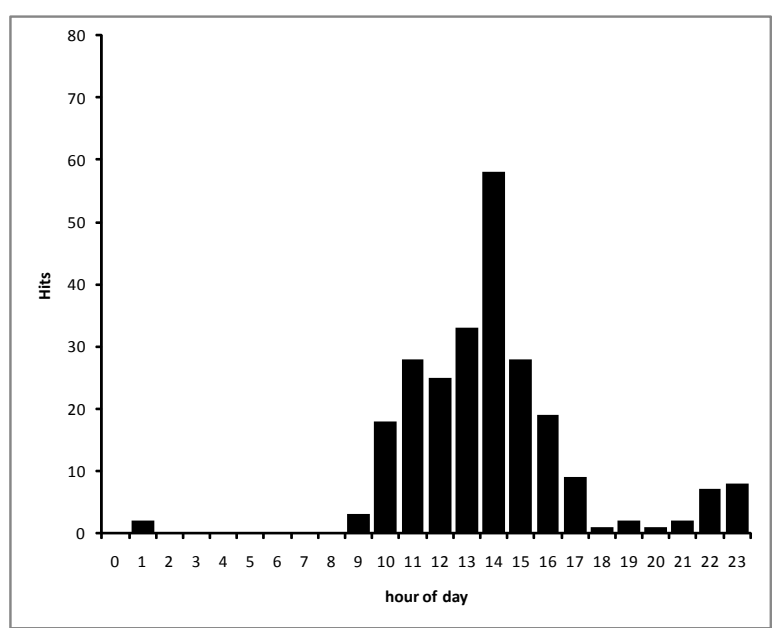

Figure 4 Time of access to lab Blackboard site by hour for student 2

The students were limited in their time in the lab by their timetables and restrictions to work under supervised conditions during normal working hours. This is largely reflected in when they accessed the wiki, though it is clear that they also accessed the system from other computers outside of the lab (see figures 3 and 4 ). It was noted that most commonly both students were in the lab on Tuesdays and Thursdays due to gaps in their timetables on these days. Again, this is reflected in their access of the Blackboard site (see figs 5 and 6 ).

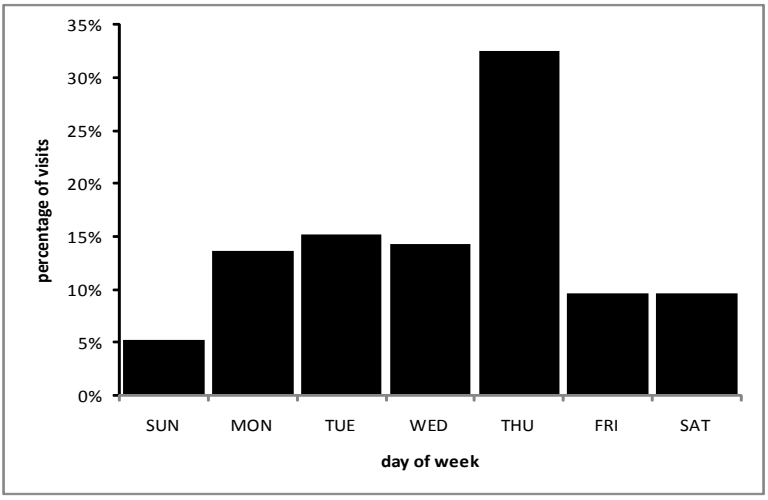

Figure 5 Access of lab Blackboard site by day of the week for student 1 (percentage of visits/ day of week)

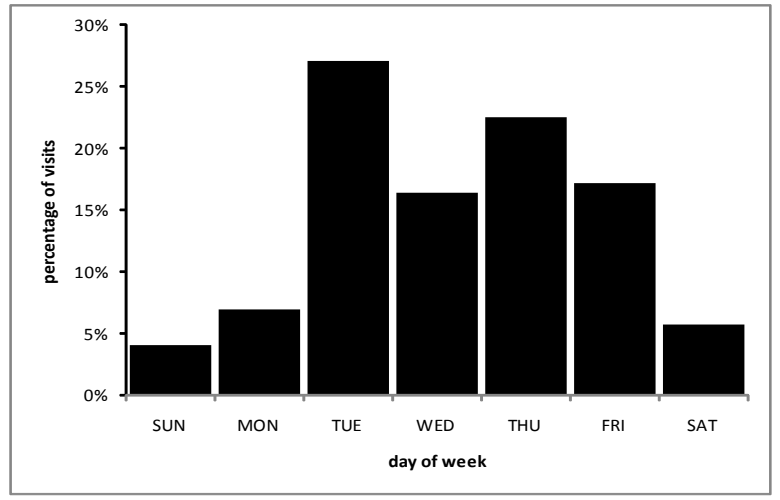

Figure 6 Access of lab Blackboard site by day of the week for student 2 (percentage of visits/ day of week)

The supervisor met with the students every week to review their progress and accessed their wiki lab books prior to each meeting. The supervisor's office was not adjacent to the lab, and so online access to the student's lab books facilitated supervision without the need for increased physical presence in the lab.

As neither of these students had carried out a research project using a recording method other than the wiki, it is impossible to compare their performance with students using traditional paper lab books. However it appeared that the students were generally more diligent in annotating gel photographs in the wiki than previous students had been when using paper copies and their methods were more clearly recorded from the very beginning of the project.

This successful experiment in supervision using electronic notebooks provided a searchable record of student work and a permanent online record of student data, which has been retained to inform future projects. Tracking student progress was facilitated by automated date and time stamping of wiki entries. The students benefitted from a significant reduction of transcription errors in writing up results, better record keeping, off campus access to and secure back-ups of their results. 


\section{Communicating author}

Dr Richard Badge, Department of Genetics, Adrian Building, University of Leicester. LE1 7RH. Email: rmb19@leicester.ac.uk, Fax: 01162525042 